\title{
NuSTAR observations of rotation-powered pulsars and magnetars
}

V. M. Kaspi ${ }^{1}$, H. An ${ }^{1}$, M. Bachetti ${ }^{2}$, E. Bellm ${ }^{3}$, A. M. Beloborodov ${ }^{4}$, S. Boggs ${ }^{5}$, D. Chakrabarty $^{6}$, F. Christensen ${ }^{7}$, B. Craig ${ }^{5,8}$, F. Dufour ${ }^{1}$, F. Harrison ${ }^{3}$, E. V. Gotthelf ${ }^{4}$, T. Kitaguchi ${ }^{9}$, C. Kouveliotou ${ }^{10}$,

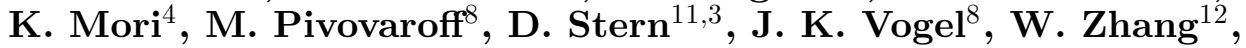
and The NuSTAR Team

\footnotetext{
${ }^{1}$ Department of Physics, McGill University, Montreal, Canada email: vkaspi@physics.mcgill.ca

${ }^{2}$ IRAP/Toulouse, France

${ }^{3}$ California Institute of Technology, USA

${ }^{4}$ Columbia University, USA

${ }^{5}$ University of California, Berkeley, USA

${ }^{6}$ Massachusetts Institute of Technology, USA

${ }^{7}$ Denmark Technical University, Denmark

${ }^{8}$ Lawrence Livermore National Laboratories, USA

${ }^{9}$ RIKEN, Japan

${ }^{10}$ NASA/Marshall Space Flight Center, USA

${ }^{11}$ Jet Propulsion Laboratory, USA

${ }^{12}$ NASA/Goddard Space Flight Center, USA
}

\begin{abstract}
NASA's NuSTAR observatory is the first focusing hard X-ray telescope. Launched in June 2012, NuSTAR is sensitive in the $3-79 \mathrm{keV}$ range with unprecedented $\sim 17^{\prime \prime}$ FWHM angular resolution above $12 \mathrm{keV}$, a result of its multilayer-coated optics and 10-m focal length. With its large effective area $\left(900 \mathrm{~cm}^{2}\right.$ at $\left.10 \mathrm{keV}\right), N u S T A R$ has point-source sensitivity $\sim 100$ times better than previous hard X-ray telescopes. Here we describe NuSTAR and its planned work on rotation-powered pulsars and magnetars during its nominal 2-yr baseline mission that has just commenced.
\end{abstract}

Keywords. stars: neutron, pulsars: general, X-rays: general, X-rays: binaries, telescopes

\section{Introduction}

The hard X-ray regime is one of the few underexplored areas of the electromagnetic spectrum in astrophysics, yet has great scientific significance. In contrast to X-rays with energies below $\sim 3 \mathrm{keV}$, hard X-rays are largely unaffected by photoelectric absorption, either due to material intervening in the Galaxy or intrinsic to the source. Thus hard $\mathrm{X}$-rays permit unobscured views to distant Galactic Plane sources, as well as to the hearts of otherwise highly obscured targets. Moreover, hard X-ray studies probe nonthermal emission regimes above where thermal X-rays confuse spectra, allowing probes of powerful particle acceleration mechanisms. Several radioactive decay lines, such as the $68-\mathrm{keV}$ line of ${ }^{44} \mathrm{Ti}$, lie in the hard X-ray range, and offer unique probes of short-lived phenomena such as supernovae.

Although of great astrophysical value, hard X-rays have been notoriously hard to focus. Previous hard X-ray telescopes such as the IBIS instrument aboard INTEGRAL 


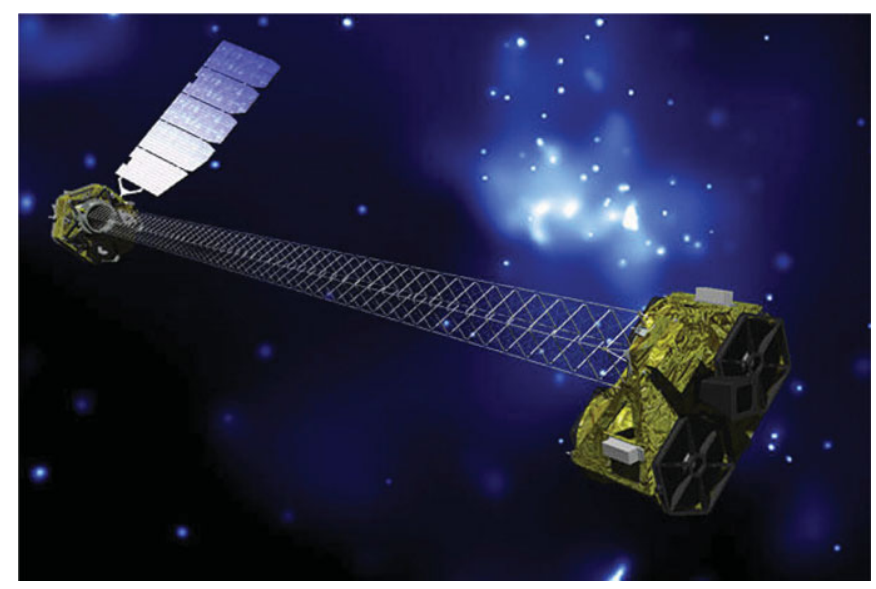

Figure 1. Artist's concept of $N u S T A R$ deployed in space.

used coded-mask technologies in which a specially patterned coded aperture mask casts a 'shadow pattern' on an X-ray detector. Deconvolution of the shadow pattern typically yielded point-spread-functions on the order of $12^{\prime}$ in the hard X-ray regime, not highly competitive with most other areas of astronomy. By contrast, NuSTAR's mirrors, arranged in a conical approximation to a standard Wolter-I type geometry, make use of newly developed depth-graded multilayer coatings that effectively Bragg-reflect incoming X-rays in the $3-79 \mathrm{keV}$ range with high grazing angles. To beef up collecting area, NuSTAR uses many nested co-axial mirrors, each specially coated and shaped. The high-energy range of the X-rays demands a $10-\mathrm{m}$ focal length, with the collected X-rays focused on CdZnTe detectors. NuSTAR has two independent mirror and detector modules, with the former separated from the latter by a 10-m-long mast. These detectors have spectral resolution of $0.9 \mathrm{keV}$ at $60 \mathrm{keV}$, and yield time resolution of $100 \mu \mathrm{s}$. The resulting mirror/detector combination yields a field of view of $13^{\prime} \times 13^{\prime}$, and a FWHM point-spread-function of $\sim 17^{\prime \prime}$. NuSTAR has a Target-of-Opportunity response time of $<48 \mathrm{hr}$. See Harrison et al. (2010) for more details of the NuSTAR mission. Figure 1 shows an artist's concept of NuSTAR deployed in space, and Figure 2 shows NuSTAR's first light observation of Cyg X-1, and the contrast of the PSF with that of the previous state-of-the-art hard X-ray instrument aboard INTEGRAL. In Figure 3 we show $N u S T A R$ 's two-telescope effective area curve, along with those of two other focusing $\mathrm{X}$ ray telescopes for comparison. Table 1 summarizes NuSTAR's sensitivity in comparison with other previous hard X-ray telescopes.

NuSTAR's 10-m mast deployed not long after the mission's flawless June 13, 2012 Pegasus launch from the Reagan Test Site on Kwajalein Atoll in the Pacific Ocean. $N u S T A R$ now sits in a $600 \mathrm{~km} \times 620 \mathrm{~km}$ orbit at $6^{\circ}$ inclination. This orbit results in an estimated 10-yr lifetime for NuSTAR, which has no consumables. Presently NuSTAR is funded only for a Principle-Investigator-led baseline 2-yr mission; however, extension

Table 1. NuSTAR's sensitivity in comparison with other hard X-ray instruments.

\begin{tabular}{|l|c|}
\hline Instrument & Sensitivity \\
\hline INTEGRAL & $\sim 0.5 \mathrm{mCrab}(20-100 \mathrm{keV})$ with $>$ Ms exposure \\
Swift (BAT) & $\sim 0.8 \mathrm{mCrab}(15-150 \mathrm{keV})$ with $>$ Ms exposure \\
NuSTAR & $\sim 0.8 \mu$ Crab $(10-40 \mathrm{keV})$ with 1 Ms exposure \\
\hline
\end{tabular}




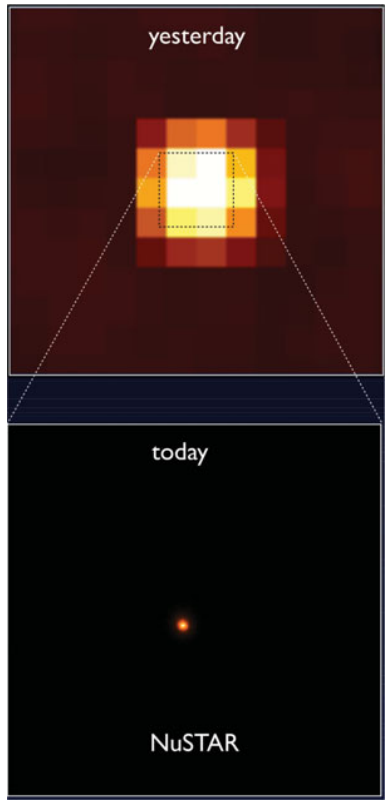

Figure 2. NuSTAR's first-light observation of Cyg X-1 (bottom) and a previous image of the same target by INTEGRAL (top), showing the dramatic improvement in angular resolution.

beyond this baseline mission and the possibility of a Guest Investigator program are currently under discussion. Following NUSTAR's calibration phase, all data obtained during the nominal 2-yr mission will be publicly available via HEASARC following a brief verification period.

\section{Current status of NuSTAR}

As of August 1, 2012, NuSTAR has completed its calibration phase and started its science operations phase. Thus far, no major anomalies have been identified. The inorbit mast motion is consistent with pre-orbit predictions. The spectral resolution is better than the pre-orbit specified requirement. The background appears to be stable and within the pre-launch requirements. The low-energy $(3-20 \mathrm{keV})$ calibration agrees with that of Swift XRT at the $<3 \%$ level. The high-energy calibration is presently under study but thus far there are no major surprises. Timing calibration is also underway; with post-facto clock-drift corrections, absolute timing at the few-ms level is expected, although for fixed pointings and short observations, the full $100-\mu$ s resolution will be usable.

\section{NuSTAR science Working Groups}

Science to be done with $N u S T A R$ has been organized into multiple Working Groups. These are, with Working Group Chair in parentheses:

- Heliophysics and Protostar Flares (D. Smith)

- Galactic Plane Survey (C. Hailey)

- Supernovae (S. Boggs)

- Supernova Remnants and Pulsar Wind Nebulae (F. Harrison) 


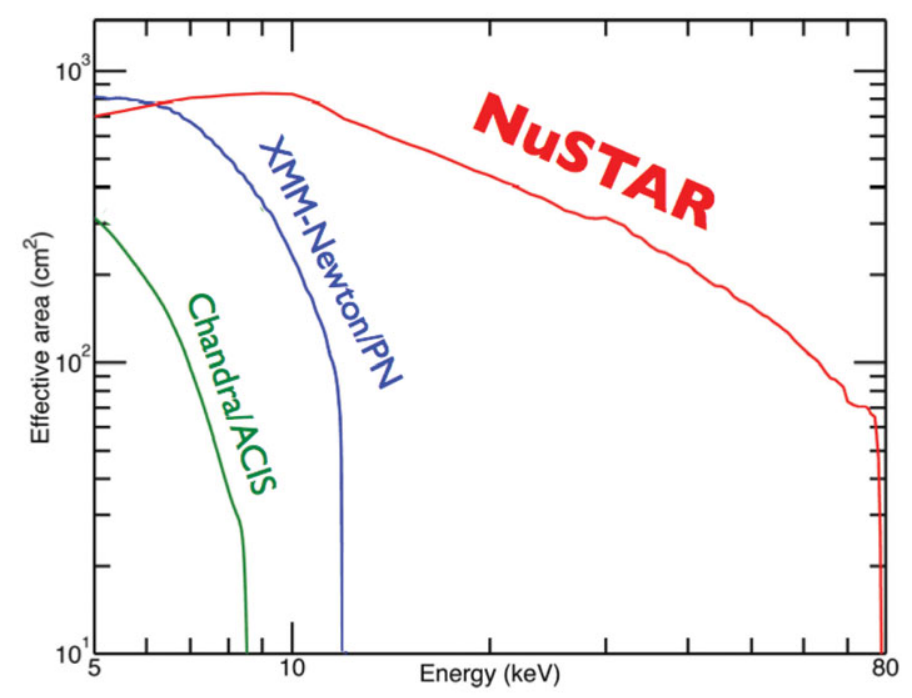

Figure 3. Effective area as a function of energy for the combination of NuSTAR's two telescopes, compared with two other focusing X-ray missions.

- Galactic Accreting Binaries (J. Tomsick)

- Magnetars and Rotation-Powered Pulsars (V. Kaspi)

- Ultraluminous X-ray Sources (F. Harrison)

- Extragalactic Surveys (D. Stern)

- Blazars and Radio Galaxies (G. Madejski)

- AGN Physics (G. Matt)

- Obscured AGN (D. Stern)

- Galaxy Clusters (A. Hornstrup, S. Molendi)

- Starburst and Local Group Galaxies (A. Hornschemeier)

A list of the membership of each of these working groups is provided at http://www.nustar.caltech.edu/for-astronomers/science-working-groups.

\section{NuSTAR Working Group on rotation-powered pulsars and magnetars}

NuSTAR's Working Group on rotation-powered pulsars and magnetars has been allocated 1.2 Ms of Priority A observing time during the nominal 2-yr mission. After careful consideration of NuSTAR's unique strengths as well as the most interesting tractable problems relevant to high-energy X-rays for rotation-powered pulsars and magnetars, our Working Group decided on the Priority A target list described below (and also available at the above-mentioned web site).

Note that the Working Group chose heavy weighting toward magnetars. This is because of the surprising high-energy turnover in their X-ray spectra as discovered by INTEGRAL and RXTE (Kuiper et al. 2006). Indeed some magnetars have more energy output above $10 \mathrm{keV}$ than below it! This emission is both unpredicted and not understood, though some models have been suggested (e.g. Heyl \& Hernquist 2005; Baring \& Harding 2007; Beloborodov \& Thompson 2007; Beloborodov 2012) and some intriguing correlations noted (Kaspi \& Boydstun 2010; Enoto et al. 2011). By contrast, many of the most energetic, hence X-ray luminous rotation-powered pulsars show simple power-law spectra from low through high X-ray energies. 
- Magnetar Target-of-Opportunity (150 ks): NuSTAR plans to observe a magnetar outburst and relaxation following a burst and/or flux trigger, provided likely by either Swift/BAT or Fermi/GBM. The goal here is to monitor simultaneously the outburst and relaxation behavior of the soft and hard X-ray components, to see whether they vary in concert both spectrally and in terms of flux. This will help constrain models for the origin of the hard component and how it is related to the observed thermal component.

- 1E 2259+586 (170 ks): This magnetar was only marginally detected in the hard X-ray band by RXTE and INTEGRAL, owing to its soft spectrum below $10 \mathrm{keV}$ and in spite of its extremely hard spectrum above (Kuiper et al. 2006). Indeed only the pulsed component was measurable with those instruments. NuSTAR will provide the first high quality broad-band X-ray spectrum for this target, which has the most extreme spectral turnover yet seen in any magnetar.

- 1E 1048-5937 (400 ks): This is the lone 'classical' regularly monitored magnetar for which no hard X-ray emission has yet been detectable. Assuming a spectral turnover in agreement with the rough correlation suggested by Kaspi \& Boydstun (2010), NuSTAR should provide the first broadband spectrum for the source, and hence a clear test of the putative correlation.

- AE Aquarii (126 ks): This target is an intermediate polar, i.e. an accreting white dwarf that has shown unique signatures of possible non-thermal X-ray emission in Suzaku observations (Terada et al. 2008). Specifically a putative sharp spike in its light curve was reported and is reminiscent of non-thermal X-ray pulses seen in rotation-powered pulsars. This is suggestive of the intriguing possibility that magnetospheric particle acceleration in this white dwarf is occuring just like that seen in rotation-powered pulsars. NuSTAR's superior point-source sensitivity will allow us to clearly test Suzaku's report. NuSTAR observed AE Aquarii in September 2012. These data are currently being analyzed.

- Geminga (260 ks): Geminga is among the very brightest gamma-ray sources known (Abdo et al. 2010) yet shows relatively faint, mainly thermal X-ray emission (e.g. Kargaltsev et al. 2005). An archetype for many other such X-ray faint/gamma-ray bright rotation-powered pulsars, NuSTAR observations can help constrain the energy at which the spectrum turns up, and whether the faint power-law emission seen at the high end of the soft X-ray band extrapolates up to the Fermi band. NuSTAR observations of Geminga were done in September 2012 and are currently under analysis. Another carefully considered target for this observation was the Vela pulsar. However, its bright and very large pulsar wind nebula yields too high a background for the pulsar to be usefully studied with NUSTAR.

- PSR J1023+0038 (100 ks): This rotation-powered pulsar is a millisecond pulsar in a 0.2-day orbit with a non-degenerate companion star that apparently had an accretion disk in the past decade (Archibald et al. 2009). X-ray emission from the system as observed with XMM and Chandra (Archibald et al. 2010; Bogdanov et al. 2011) shows a hard power-law tail suggesting detectability well above $10 \mathrm{keV}$ with $N u S T A R$. This system is a unique transition object between the millisecond pulsar and low-mass X-ray binary phases of binary evolution. As such, it can further serve as a comparison via $N u S T A R$ observed spectral and timing behavior with current quiescent low-mass X-ray binaries that show similar hard X-ray power laws. The latter have been hypothesized to indicate the presence of unseen millisecond radio pulsars which become active when the X-ray binary is in its quiescent state (e.g. Burderi et al. 2003). 
- 1E 1841-045 (45 ks): This target is a magnetar located at the center of the supernova remnant Kes 73. It was among the first magnetars noted by Kuiper et al. (2006) to be a hard X-ray emitter. NuSTAR, with a relatively short exposure, can measure an exquisitely precise spectrum, clearly defining the turnover region as well as the pulsed fraction as a function of energy. This target's emission will also serve as a sanity check for comparison with previous measurements, and coupled with other such comparison observations using other objects, can check for variability in this source.

\section{Conclusions}

NuSTAR's superb capabilities and flawless launch made this an exciting time in highenergy astrophysics. The telescope is poised to tackle a wide range of astrophysics with revolutionary angular and spectral resolution in its energy range. For rotation-powered pulsars and magnetars, NuSTAR will be observing some of the most interesting objects known, and tackling problems that have great relevance to a wide class of astrophysical sources in high-energy astrophysics. Please stay tuned for these upcoming science results, which should be forthcoming in early 2013, once $N u S T A R$ 's basic calibration is complete and these unprecedented data sets are analyzed.

\section{References}

Abdo, A. A., et al. 2010, ApJ, 720, 272

Archibald, A. M., et al. 2009, Science, 324, 1411

Archibald, A. M., et al. 2010, ApJ, 722, 88

Baring, M. G. \& Harding, A. K. 2007, ApJSS, 308, 109

Beloborodov, A. M. 2012, ApJ, in press, arXiv:1201.0664

Beloborodov, A. M. \& Thompson, C. 2007, ApJ, 657, 967

Bogdanov, S., et al. 2011, ApJ, 742, 97

Burderi, L., et al. 2003, A\&A, 404, L43

Enoto, R., et al. 2011, ApJ, 63, 387

Harrison, F. A., et al. 2010, SPIE, 7732, 77320S

Heyl, J. \& Hernquist, L. 2005, ApJ, 618, 463

Kargaltsev, O. Y., Pavlov, G. G., Zavlin, V. E., Romani, R. W. 2005 ApJ, 625, 307

Kaspi, V. M. \& Boydstun, K. 2010, ApJ, 710, L115

Kuiper, L., Hermsen, W., den Hartog, P. R., \& Collmar, W. 2006, ApJ, 645, 556

Terada, Y., et al. 2008, PASJ, 60, 387 